\title{
Carnets
}

Revue électronique d'études françaises de l'APEF

Deuxième série - $18 \mid 2020$

Chiens et écritures (littéraires, filmiques, photographiques)

\section{William Wegman : un artiste dans un jeu de quilles}

Le chien acteur d'une déconstruction iconique

\section{Vincent Lecomte}

\section{(2) OpenEdition}

\section{Journals}

Édition électronique

URL : http://journals.openedition.org/carnets/10548

DOI : $10.4000 /$ carnets. 10548

ISSN : 1646-7698

Éditeur

APEF

Référence électronique

Vincent Lecomte, "William Wegman : un artiste dans un jeu de quilles », Carnets [En ligne], Deuxième série - 18 | 2020, mis en ligne le 31 janvier 2020, consulté le 04 juin 2020. URL : http:// journals.openedition.org/carnets/10548; DOI : https://doi.org/10.4000/carnets.10548

Ce document a été généré automatiquement le 4 juin 2020

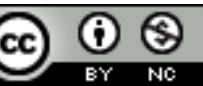

Carnets est mis à disposition selon les termes de la licence Creative Commons - Atribution - Pas d'utilisation commerciale 4.0 International. 


\title{
William Wegman : un artiste dans un jeu de quilles
}

Le chien acteur d'une déconstruction iconique

\author{
Vincent Lecomte
}

\section{Ni chien ni maître}

1 De nombreux artistes ou penseurs ont eu une relation particulière avec leur chien. À commencer par René Descartes qui, bien que père du concept d'animal-machine, vouait à son chien, Monsieur Grat, la plus grande affection ${ }^{1}$. Nicolas Malebranche, à la fin du XVII ${ }^{\mathrm{e}}$ siècle, décrit, dans ses Entretiens sur la métaphysique et la religion, l'étroite relation qu'il établit avec son chien, et l'esquisse de dialogue qu'il sent naître à son contact (Malebranche, 1948: 110). Toutefois, le philosophe affirme que s'il existe bien une communication naturelle entre lui et l'animal, et des rouages émotionnels similaires et concordants, contrairement à l'homme, doué d'âme, le chien n'est que «pure machine » (ibid.). Plus tard, Arthur Schopenhauer affirmera : s'il n'y avait pas de chiens, je ne pourrais pas vivre ${ }^{2}$. Pour le philosophe allemand, la compagnie et plus encore l'esprit d'Atma (qui signifie en sanscrit «âme du monde ») était indispensable ${ }^{3}$. Dans Maître et chien, une idylle, Thomas Mann relate en détail les promenades qu'il fait avec Bauschan. Cet animal « bon, doux et intelligent» (ce sont les mots de l'écrivain) (Mann, $2001: 8)$, est pour lui autant un réconfort moral qu'un objet d'observation. Mais, bien que sa personnalité soit décrite avec précision, Bauschan reste un Hühnerhund, un chasseur de poules, un être définitivement inférieur, dépendant, soumis.

Bien avant les travaux photographiques de John Stortz prenant pour modèle son Husky blanc qui l'accompagne dans ses voyages et les séries Under-dogs d'Andrius $\mathrm{Burba}^{4}$, William Wegman est l'un des premiers artistes à faire d'un animal de compagnie le centre de toute son œuvre. C'est une rencontre fortuite qui est à l'origine des travaux désormais les plus connus de l'artiste 5 . Dans l'atelier qu'il occupe en Californie, Wegman se retrouve dès lors flanqué d'un braque de Weimar, qui lui tient d'abord simplement compagnie. Il remarque rapidement que ce chien est très joueur, 
mais aussi qu'il s'ennuie dans cet espace exigu. Alors que l'animal traverse intempestivement le champ de son objectif, l'artiste perçoit soudain l'occasion qui se présente d'une recherche esthétique inédite. C'est le début d'une longue collaboration, qui revêtira même, avec le succès, un caractère emblématique. Wegman lui-même en convient : « pour le meilleur comme pour le pire, je suis l'homme au chien ${ }^{6}$ ». Mais, bien que centré sur un sujet privilégié, son travail reste néanmoins varié, voire polymorphe : au-delà de la photographie, il inclut le dessin, la peinture, le collage et surtout la vidéo.

3 L'artiste repère rapidement dans son premier modèle canin, âgé de six semaines, un «potentiel photogénique ». Mais, réussir à capter l'attention du chien est soit l'effet du hasard, soit le résultat d'une stratégie déployée afin de stimuler ses instincts ou ses intérêts. Si la prise de vue proprement dite est instantanée, la préparation est longue. Peu à peu va s'engager entre l'artiste et le chiot une connaissance ou reconnaissance respective, une sorte de domestication réciproque, d'un genre nouveau.

\section{Jeux de chiens, jeux de malins}

4 De manière parallèle, les jeux que Wegman met en place répondent de plus en plus aux dispositions du braque, qui, en miroir, apprend à répondre et à devancer les demandes. L'artiste privilégie parfois l'image animée, plus à même de rendre fidèlement la dynamique animale qui semble faire défaut aux poses statiques obtenues sur commande. Mais la saisie du mouvement, son rendu photographique, le «bougé», qu'on retrouve dans diverses séries, arrive également à la transmettre. Il est à noter que si l'animal constitue couramment le sujet unique, l'homme, pourtant invisible, est omniprésent. Dans la série des Flying dogs, initiée en 2010, la capture du mouvement représente la part essentielle de ce travail, mais l'origine humaine de la propulsion, et de cette chorégraphie aérienne, donnant vie à ces « chiens volants ${ }^{7}$ ", est évidente.

5 La démarche canine est elle aussi revisitée de manière humoristique. La série des Stormy night de 1972 semble modelée sur les études photographiques d'Eadweard Muybridge ${ }^{8}$. À l'instar des travaux du chronophotographe, le pas est décomposé, la posture du sujet précisément analysée, tandis qu'un simple cube blanc, sorte de piédestal, offre soudain une lecture à l'antique de l'étude, replaçant la figuration du corps animal dans l'héritage de la statuaire. Cette dernière trouvera d'ailleurs l'année suivante sa version moderne dans Ray-o-vac, photographie dont le titre est tiré d'une célèbre marque de piles. L'énergie canine sert cette fois une exploration du mobilier informatique. Le photographe dans cette mise en scène déjoue les codes esthétiques en les télescopant: bel objet ménager / beau chien de compagnie. Il rend également hommage à cet imaginaire surréaliste de l'incongru et des jeux de mots et de formes.

Chez Wegman, on peut remarquer un désir récurrent de se référer à l'histoire de l'art, ou plus généralement à ce qu'on pourrait nommer un "bain iconique ». Comme Man Ray - il donnera d'ailleurs à son premier braque le nom de l'artiste -, Wegman est fasciné par le champ publicitaire. Qui plus est, ce chien gravissant cet escalier fait d'ustensiles n'est-il pas, à sa façon, un nu remontant l'escalier', référence à Marcel Duchamp, en forme d'écho, de reflet ou simplement de réponse ? Substituer un chien à un modèle féminin, une " pin-up ", ne donne-t-il pas aussi à penser un certain pouvoir d'attraction, fondé sur tout autre chose que l'utilitaire? 
7 L'animal, comme statufié, chevauche fièrement les emblèmes de la sophistication technique. Le chien paraît, dans une attitude souveraine et sereine, relativiser, placidement, la perfection de ces produits et, partant, de l'intelligence humaine ${ }^{10}$. Le plaisir du jeu et l'ironie sont présents dans toute l'œuvre de Wegman, incitant le spectateur à déchiffrer les codes utilisés. Tel un personnage type, la figure animale, centrale, facilite l'accès à cet univers référentiel. Les situations, postures, habits, décors appartiennent tous à l'humain; le chien en propose une relecture animale ironique.

\section{Le corps, l'objet}

8 Investies par ce corps canin, ce sont soudain les figures d'un imaginaire humain qui se voient éloignées, caricaturées, objectivées. Dans ce processus, les intentions posturales laissent saisir une forme de pétrification ${ }^{11}$. Dans cette immense galerie de portraits, d'image en image, de série en série, de film en film, d'émission en émission, le regard reste suspendu, balançant entre identification impossible par la nature même du sujet, et affinité avec un modèle et un univers aisément reconnaissables. La légèreté et l'inconséquence apparentes du jeu permettent une forme d'expression de soi difficile à atteindre par d'autres moyens, rendue ici possible par la simple présentation du corps animal.

Lorsque le corps du chien est habillé, l'on pourrait croire avoir affaire à un personnage cynocéphale, rappelant l'iconographie héraldique ou légendaire, voire l'image d'Épinal ${ }^{12}$. On peut encore songer aux illustrations de Grandville, aux collages de Max Ernst ou aux photomontages de John Heartfield ${ }^{13}$. Dans la lignée de cet héritage, Wegman réalise, entre autres, en 2007, un triptyque intitulé Hansel and Gretel ${ }^{14}$, représentant un couple de chiens dont seules les têtes sont visibles, le reste du corps étant recouvert d'une chemise et d'un pantalon blanc crème. Si le photographe s'inscrit dans une veine souvent satirique, la présence d'un véritable chien confère à son travail une puissance d'identification que les animaux humanisés jusqu'alors ne possédaient pas - ou alors d'une manière superficielle, allusive, incidente.

10 Par ailleurs, le fait qu'il s'agisse toujours du même animal - du moins en apparence, car Wegman remplace son modèle, lorsqu'il meurt, par un autre braque, finissant même par en posséder plusieurs en même temps - permet au spectateur une reconnaissance immédiate de l'auteur. Le caractère atemporel, éternel de ses sujets, semblant jouir d'une vie défiant le temps et toute chronologie, est une des raisons pour lesquelles l'on peut persister à évoquer la notion de «figure » à propos de l'emploi de ces chiens. Car il s'agit bien là d'une véritable forme type, qui est capable de révéler et de marquer un style, mais aussi de produire un réseau d'images fonctionnant en système interdépendant, voire inter et autoréférencé.

11 Par la substitution systématique du corps de l'animal à celui de l'homme, l'artiste revisite inlassablement les références classiques, les icônes, et même les récits qui forment la représentation qu'une part importante des humains se fait du monde, du vivant et d'elle-même. C'est également l'« œuvre » d'une réaction anthropomorphique qui est comme prise au piège.

12 Devenus supports, motifs, figures de jeux - mais jamais réduits au statut de jouet -, les chiens, au sein des stratégies toujours renouvelées de Wegman, semblent accéder au rang d'icône, sans pour autant perdre de leur présence temporelle. Pourtant, ces 
animaux sont-ils encore, sur cette scène, des «existants»? Leur corps au pelage soyeux, gris ou marron, l'allure fière de leur silhouette sont magnifiés par une esthétique photographique de studio, et une pratique qui les prend comme sujets exclusifs et inépuisables. Wegman, dans sa direction d'acteur, pourrait-on dire, va du souci esthétique, portant sur la "plasticité " physique, à l'attention éthologique au vécu et aux réactions de ses modèles.

\section{Un théâtre canin} beaucoup dans l'efficace et dans la force de l'œuvre du photographe. Wegman ne cesse pas d'être présent à travers les mises en scène, les costumes choisis, les angles de prise de vue, les postures des chiens... Leur corps, qui rappelle constamment le comportement humain, semble exprimer un vouloir humain. Il est certes difficile pour la plupart des spectateurs de confondre les désirs d'un homme avec ceux d'un animal. Toutefois, une forme d'indistinction psychologique semble entretenue, voire cultivée par le photographe. Seulement, le chien, sans perdre sa personnalité ${ }^{16}$, ne se réduit pas 
à un simple pantin que l'on pourrait faire bouger, objectivant un inconscient individuel ou collectif.

Il s'agit bien ici de jeu, celui de comédiens canins. Mais on peut dire, aussi et surtout, que Wegman se joue de l'espace scénique et des astuces que permet la mise en scène. "Starifiés» depuis des années, les braques finissent même par prendre place, subrepticement, au sein d'un panthéon de figures médiatiques. L'artiste rappelle ainsi que «Man Ray, décédé en 1982, a été élu 'homme de l'année' par Village Voice.» (Delcourt, 2017 : 30) Paradoxe second : s'ils sont des stars, les chiens finissent par ne plus représenter, désigner qu'eux-mêmes, dans les œuvres de Wegman. Les rôles qu'ils incarnent disparaissent au profit du surgissement de leur présence animale, mais aussi de leur individualité. C'est eux « en acteurs » qu'on attend. Et, en quelque sorte, ce qui, sur cette scène parallèle, joue le rôle central est bel et bien ce que l'on appelle communément la «fibre sentimentale ». Brigitte Ollier perçoit dans le travail presque monomaniaque du photographe un «cocktail d'humain et de canin, d'humour et d'amour, (...) à la lisière de Walt Disney, de Marcel Duchamp et de Buster Keaton... » (Ollier, 1996)

19 L'héritage des documentaires animaliers, et, notamment, ceux des studios Disney ${ }^{17}$, présents, de longues années durant, sur les téléviseurs de nombreux pays, est indéniable. Dans ces films, l'humanisation est opérante à travers une voix off omniprésente, une scénographie de la vie animale, donnée à voir suivant des comportements, des intentions et même une conscience humaines ${ }^{18}$. Wegman joue de cette «filiation " pour mieux la contrarier. Il la déjoue plutôt, en en reprenant sans retenue le principe et en en inversant le processus. L'animalisation de l'homme remplace alors l'humanisation de l'animal.

20 Ces mises en scènes révèlent principalement une volonté de se saisir du corps de l'animal pour réussir à évoquer (et provoquer) celui de l'homme. Mais le corps de l'animal, par-delà même l'imaginaire qui lui est associé, ouvre sur un ailleurs qui pourtant est un monde proche de celui de l'homme, voire confondu au sien. Dans les séries télévisées qui ont accompagné l'enfance de Wegman, comme Rintintin ou Lassie ${ }^{19}$, le chien -protagoniste principal dont le comportement avisé fera basculer l'action -, possède comme une psychologie humaine, ou en accord avec elle. Si Wegman ne va pas jusqu'à montrer l'intimité animale de ses chiens, il ne propose pas pour autant une vision aussi benoîtement morale du comportement animal. Et sa présence de metteur en scène, qui ne peut échapper à l'observateur, est l'une des caractéristiques principales de son œuvre. Il n'y a pas de réconciliation avec la nature, comme on peut l'observer par exemple chez Rudyard Kipling, mais la charge affective n'est pas pour autant éludée.

21 La complicité avec le modèle canin, doublée d'une attention soutenue et permanente de la part de Wegman, démontre son désir de donner une place moins subalterne, ou accessoire, au chien dans la société. La force anthropomorphique qui hante son travail finit par servir une véritable critique de ce sentiment qui conditionne profondément notre rapport à l'animal. S'estompe peu à peu, dans la perception de l'artiste, mais également dans celle qu'il attribue à son acteur-modèle, la précision des frontières qui s'établissaient entre l'animal et l'homme. Il offre à voir une sorte de réalité « neutre »: une conscience du vivant, commune. Cette conscience animale peut finir par émerger tel un phénomène global, essentiel, une quiddité, dont la « canidité » ne serait qu'une version parmi d'autres. L'animal «atteint »l'homme également d'une autre façon. Le 
chien, comme bien d'autres animaux, devient la promesse d'une révolution ontologique.

En présence du corps animal, on s'aperçoit que l'humanité comme la canidité sont des leurres, des fantasmes d'essentialité ${ }^{20}$. L'apparition animale sur la scène humaine a valeur de révélation: cette humanité n'est pas une extériorisation de l'Esprit. Pour Schopenhauer, «le monde n'est que la connaissance de soi de la Volonté» (Schopenhauer, $1926: 723$ ) que le corps de tout animal agit.

\section{Se jouer pour déjouer}

Wegman met à jour un être pensant. Lolita, et son " regard-caméra », reste l'un des exemples saillants de cette percée de la conscience. Avec Wegman, l'animal de foire, le "chien savant», réinvente ce théâtre de l'intérieur, par son intériorité soudain révélée ${ }^{21}$.

Avec une œuvre telle que Lolita (1990), l'artiste se dirige vers un registre sensuel. Le chien est ici présenté sans accessoire ni vêtement; son corps envahit l'espace photographié, serait-on tenté de dire, dans son plus simple appareil. Mais c'est sa posture qui crée le trouble. En effet, «avachi » sur un siège pliant rudimentaire et se détachant sur un fond marron rehaussé de rouge, le corps de la bête évoque l'allure d'une jeune fille alanguie, et qui s'offrirait complaisamment au regard qui vient capturer ce moment d'abandon. La chienne a les yeux fixés sur l'objectif ; ils sont peu expressifs, mais ce ne sont toutefois pas ceux d'un animal désintéressé par une activité humaine qui ne le concernerait pas. Ce regard vide semble bien plutôt hanté par la désinvolture d'une personne consciente de son effet, faisant semblant de l'ignorer, exacerbant par cette attitude le désir qu'elle sait pertinemment provoquer.

Cette fascination pour et par l'aura d'un sujet offert au regard d'un spectateur-voyeur, c'est ce que provoque précisément le personnage éponyme du roman de Vladimir Nabokov $^{22}$. Il s'agit encore une fois pour Wegman de se placer dans la position de l'exégète d'une œuvre, de telle sorte que le ludique, complice ici du lubrique, rabat, dans un procédé héroïco-comique, les codes et les images mythiques au rang de l'animal. L'exhibition d'une corporalité ne connaissant pas de honte, plus encore qu'un nouvel écran, est la réincarnation du personnage. Sous le poil soyeux et ras, la chair est présente dans sa pleine puissance et dans le pur déploiement de sa sensualité.

Avec Lolita, l'observateur hésite soudain entre le modèle canin, à la présence irréfutable, et une multitude d'images à connotation érotique. Pris dans un piège spéculaire, le spectateur voit ressurgir l'animalité, non plus dans la photographie, mais en lui. Le corps avachi de l'animal, tel celui d'un modèle désirable, semblerait presque pouvoir éveiller l'impossible: la possibilité, effrayante, d'une forme d'attirance zoophile. La présence de l'animal objective le jeu des pulsions, provoquant un étrange sursaut rationnel. Mais la raison - comme surprise dans une fragilité inattendue - ne peut étouffer complètement l'attraction première.

\section{Portrait de l'homme en chien : jeu de regards}

Chez Aristote, l'homme ne s'extrait pas du monde vivant, même s'il en constitue l'achèvement. Il n'en reste pas moins « animal politique ${ }^{23}$ ». Wegman, dans son sillage, à 
travers ces images ironiques, faussement attendrissantes et souvent provocatrices, forme une galerie de portraits de l'homme en animal. Ici, c'est bien cette polis, principale preuve de la singularité extra- ou sur-animale de l'humain, qui est la cible.

De fait, l'anthropomorphisme n'est plus subi mais utilisé et mis en scène, afin de faire comprendre ses ressorts. Le chien, participant au délitement, voire à la destruction du métaphorique, vient donner plus qu'un corps à cette incarnation de l'animal politique. Wegman prend dans la vaste bibliothèque des idées et images la matière d'une réflexion iconique sur les modes d'être de l'homme, et sa manière de prendre place dans le monde.

Chez Wegman, le sourire possède une vertu salutaire, facilitant un transfert projectif décomplexé. Substituant au modèle humain un chien, les codes du portrait se trouvent revisités; il s'agit de «s'explorer » en suivant les études de la représentation humaine, et de sonder les attentes, les désirs qui hantent une société d'images. C'est finalement l'entièreté d'une forme de conditionnement iconique qui est ici visée. Et c'est le déplacement, le décentrement par le chien qui permet cette révélation. L'humour oscille entre la provocation et l'ironie, et, passé un élan affectif premier, comme augmenté par la figure du chien, le spectateur se trouve dans un temps second face à la critique de ce qui "va de soi", de l'évidence du modèle humain. Semblent ainsi se métaphoriser les systèmes comportementaux de l'homme. Une série datant de 2017 porte d'ailleurs un titre qu'il serait peut-être bon de prendre au pied de la lettre : Being human.

Dans nombre de travaux photographiques, les personnages animaux de Wegman se détachent sur des fonds unis, les accessoires et vêtements sont mis en valeur tels des éléments symboliques; le chien devient le dépositaire d'une mémoire de notre propre espèce. L'inconscient culturel, sollicité à travers un symbolisme épuré, délivre une sorte de paradigme de l'humanité qui finit par servir le portrait de l'homme. On le reconnaît alors par ses faiblesses, sa violence, ses désirs, ses peurs... Dans ce jeu de rôles et de caractères, le chien, vecteur de sens, devient le point de vue extérieur idéal porté sur nos comportements et fonctionnements sociaux. Il représente une présence spéculaire, et sa distance "spécifique " permet une analyse critique de l'animal humain. Ce jeu de miroir est réciproque : le chien renvoie notre image revisitée par le corps canin, mais encore, sa figuration est un constant rappel de la relation que nous entretenons avec lui.

31 Le regard que l'animal porte sur nous, ou du moins ce qu'il nous en laisse deviner, ouvre sur un monde que nous avons par définition en commun - même si nous en refusons bien souvent le partage, et si celui-ci garde un caractère imprécis ou incertain. La «pensivité » que ses photographies viennent révéler ici n'est pas sans rappeler celle que Jacques Derrida perçoit, au sortir de la douche, dans l'œil de son chat ${ }^{24}$ et dont Jean-Christophe Bailly témoigne dans Le Versant animal, à l'occasion de la rencontre fortuite d'un chevreuil. Pour Bailly, «la pensivité des animaux (...) n'est ni divertissement ni curiosité : ce qu'elle établit c'est que le monde où nous vivons est regardé par d'autres êtres, c'est qu'il y a un partage du visible. » (Bailly, 2001 : 33)

Jouer avec ses chiens pour donner vie à une œuvre cohérente, intrigante, pensante, c'est pour Wegman activer inlassablement une mécanique de personnification, d'individuation - étrangère à l'homme bien qu'il en soit le sujet constant. À propos de son premier complice, l'artiste confie : « [il] n'a jamais pensé qu'il était né chien, il était d'un grand stoïcisme, et, pour moi, ce fut une découverte formelle ${ }^{25}$.» Thomas Mann, 
dans un rapprochement énigmatique, évoque, en parlant de son chien, « l'idéalisme de son instinct» (Mann, 2001 : 19). La prise en compte de la psychologie particulière de chaque chien - que Wegman ne cherche pas à nier, mais sur laquelle, au contraire, il s'appuie - rend possible un dialogue entre deux êtres d'espèces différentes ${ }^{26}$.

Derrière la figure, subsiste toujours un sujet identifié ${ }^{27}$, un partenaire, un complice pris dans une subtile coévolution dont l'origine, encore incertaine, est sans doute à chercher à la fin du paléolithique ${ }^{28}$. L'artiste et ses chiens réinterrogent cette obscure et pourtant solide complicité selon des règles du jeu établies en commun.

D'après l'ethnologue Jean-Pierre Digard, il est trop fréquemment tenu pour acquis que l'homme a domestiqué l'animal pour répondre uniquement à des besoins ${ }^{29}$. Ce faisant, on oublie trop souvent une part essentielle de la relation qui s'est établie durablement entre l'homme et les autres animaux. Il faut chercher cette motivation dans la nature même de l'homme. En effet, Digard écrit : «l'homme a domestiqué, domestique encore aujourd'hui les animaux avant tout pour satisfaire son besoin intellectuel de connaissance et sa compulsion, mégalomaniaque, de domination et d'appropriation des êtres vivants ${ }^{30}$.» (Digard, 1999 : 108) Cette pulsion dominatrice et possessive (qui peut d'ailleurs s'exercer sur d'autres êtres humains) semble répondre à ce qui est conçu comme un impératif vital autant qu'éthique. Le jeu avec l'animal répond également à ce désir de savoir. On sait les vertus formatrices du jeu. Wegman, avec la complicité de ses chiens, entend initier ou réinitialiser le regard de son public.

Wegman n'hésite pas à retourner sur l'homme le miroir de ses contradictions, à commencer par celles qui caractérisent sa relation avec les autres animaux. Cependant l'artiste n'aborde jamais frontalement ce que le philosophe Gary L. Francione nomme une "schizophrénie morale ${ }^{31}$ ", qui caractérise les relations ambiguës, voire contradictoires que l'homme établit avec les autres animaux. Ce qui est à saisir avec Wegman c'est le reflet d'un être qui n'est ni tout à fait identique, ni tout à fait différent ${ }^{32}$.

36 Si pour Merleau-Ponty «l'homme n'est pas animalité » (Merleau-Ponty, 1995 : 269), c'est-à-dire que sa nature l'extrait radicalement du reste du vivant, il a, nécessairement, une connaissance de l'animal, puisqu'il en est un lui-même. Ce savoir animal n'est ici qu'esquissé par son caractère figural et ironique, dans une galerie de portraits qui tient l'homme à distance.

37 L'artiste ne nie pas la fonction transitionnelle de l'animal ; au contraire il en exacerbe la nécessité. Faisant entrer le chien dans son atelier, puis, en le plaçant au centre de cette œuvre devenue rapidement médiatique, il le fait pénétrer dans le monde de l'art. Se rejoue, de pièce en pièce, le théâtre d'une coévolution ${ }^{33}$ dont Wegman semble vouloir obstinément ressaisir la force fondatrice, face à une humanité égarée dans la profusion et la confusion des images.

Le chien redevient à la fois le référent fidèle, le gardien du foyer, veillant sur les habitus, et le trait d'union entre l'humanité et le reste de l'animalité. Peut-être de ce fait est-il condamné à errer entre les règnes. Dans ce zwischen land ${ }^{34}$, ce monde transitoire, vers lequel Wegman conduit - presque négligemment, presque puérilement - son public, l'artiste ne questionne-t-il pas inlassablement une force figurale ancestrale? 


\section{Mondo cane : jouer avec l'homme}

On l'a vu, au-delà d'un geste anthropomorphiste manifeste, l'œuvre, ou le jeu, de Wegman repose principalement sur une forme d'empathie. Si cette dernière est le vecteur d'une réflexion sur l'homme et son conditionnement social, elle peut aussi avoir une autre signification. L'empathie puise au plus profond de l'émotion, dans ce qui constitue l'homme. Pour certains sociologues et préhistoriens, elle va même jusqu'à représenter l'un des facteurs les plus «spécifiants » de l'humanisation ${ }^{35}$.

Tout comme l'enfant, par le jeu, tente de relire l'histoire des adultes afin d'en comprendre le déroulé, la raison d'être, d'en percer le mystère, jouer avec l'animal peut aider à comprendre à quoi «joue»l'homme. À notre époque, la figure animale peut servir à remettre en cause l'attitude impérialiste et violente d'une société globalisée régie par le principe paradoxal de l'exacerbation et de la dissolution de l'individu. Convoquer l'animal reviendrait à évoquer ce qu'est l'essence du vivant. Plus singulièrement, le chien que cherche à devenir l'artiste russe Oleg Kulik, dans une revendication à la fois identitaire et naturaliste, ou que laisse mourir le nicaraguayen Guillermo Vargas, pour dévoiler la cruelle ambivalence de notre rapport à l'animal, ou encore celui avec lequel semble seulement jouer William Wegman, ne participe-t-il pas de la même attitude critique envers une sauvagerie civilisationnelle - celle d'une société justement ou injustement nommée «monde de chien» - ayant anéanti toute réelle altérité?

\section{BIBLIOGRAPHIE}

ARISTOTE (1999). Politique I (trad. Pellegrin). Paris : Flammarion.

AUBERGER, Janick (2007). « Entre l'écrit et l'image, l'animal de fiction, un homme travesti ?», Contre-jour : cahiers littéraires, 13, pp. 134-147.

BAILLY, Jean-Christophe (2001). Le Versant animal. Paris : Bayard.

BETTELHeIM, Bruno (1976). Psychanalyse des contes de fées. Paris : Pocket.

BRUSSE, Julien (2009). Le Problème de l'essence de l'homme chez Spinoza. Paris : Publications de la Sorbonne.

CHRISTEN, Yves (2013). L'Animal est-il un philosophe? Poussins kantiens, bonobos aristotéliciens. Paris : Odile Jacob.

DELCOURT, Maxime (mai-juin 2017). « William Wegman », Fisheye n 24.

DIDEROT, Denis (1830). Paradoxe sur le comédien [1773]. Paris : Sautelet.

DIGARD, Jean-Pierre (1999). Les Français et leurs animaux. Paris: Fayard.

FRANCIONE, Gary L. (2000). Introduction to Animal Rights: Your Child or the Dog? Philadelphie: Temple University Press. 
FREUD, Sigmund (1985). « Lettre du 21 septembre 1897 », The Complete Letters of Sigmund Freud to Wilhelm Fliess, 1887-1904. Londres : Harvard University Press.

HUSSERL, Edmund (1973). Husserliana, Zur Phänomenologie der Intersubjektivität (Sur la Phénoménologie de l'intersubjectivité) 1905-1920. La Haye : Kern.

HUSSERL, Edmund (1995). « Le monde et nous. Le monde environnant des hommes et des bêtes » [1934], Alter n 3, L'Animal.

LESTEL, Dominique (2001). Les Origines animales de la culture. Paris : Flammarion.

MERLEAU-PONTY, Maurice (1995). La Nature, Cours du Collège de France. Paris : Seuil.

MAGGIORI, Robert (2004). « Pauvres machines à vivre ». Paris : Libération (actualité le 04 /08/2004)

[disponible le 27/04/2018] <URL : https://www.liberation.fr/ >.

MALEBRANCHE, Nicolas (1948). Entretiens sur la métaphysique et la religion, t. 2, XII. Paris : Vrin.

MANN, Thomas (2001). Maître et chien, une idylle (Herr und Hund, ein Idyll) [1918]. Paris : Livre de poche.

MANN, Thomas (2002). Considérations d'un apolitique (Betrachtungen eines Unpolitischen). Paris : Grasset.

NАBOKOV, Vladimir (1955). Lolita. Paris: Olympia Press.

OLLIER, Brigitte (1996). «William Wegman photographie ses chiens et loue leurs formes, leur 'plasticité' ». Paris : Libération. (actualité le 06/07/1996) [disponible le 17/03/2018] <URL : https://www.liberation.fr/ >.

SCHOPENHAUER, Arthur (1926). Schopenhauers Briefe an Becker, Frauenstädt, v. Doss, Lindner und Asher aus den Jahren 1813 bis 1860. Stuttgart: P. Reclam.

SPIKINS, Penny, RUTHERFORD, Holly \& NEEDHAM, Andy (novembre 2010). « From hominity to humanity: Compassion from the earliest archaic humans ». Oxford: Time et Mind, vol. 3.

\section{NOTES}

1. Il « l'aimait beaucoup, était très attentif à sa santé, se glorifiait que ses chiots fussent si beaux, et passait beaucoup de temps à batifoler avec lui. Il est certain qu'il ne l'a jamais pris pour une horloge, un treuil ou une pompe hydraulique. » (Maggiori, 2004).

2. «Wenn es keine Hunde gäbe, möchte ich nicht leben» (Schopenhauer, 1926: 46). Le philosophe aurait également affirmé, dans une formule proche de celle de Madame de Staël, et anticipant celle, plus proche encore, de Mark Twain : «plus je connais les hommes, plus j'aime mon chien ».

3. Même si celui-ci s'incarna dans de nombreux caniches qui prirent tous le même nom.

4. John Stortz prenant d'innombrables clichés de Wolfgang, qui l'accompagne dans ses voyages autour du monde. Andrius Burba dans les séries Under-dogs et Under-cats, photographie du dessous chiens et chats posés sur une vitre, se jouant de l'attendrissement pour certains animaux domestiques

5. Il s'était tout d'abord montré plutôt défavorable à l'achat d'un chien, et c'est l'insistance de sa femme qui l'a finalement conduit à y céder.

6. Propos de l'artiste recueillis par Brigitte Ollier, Libération du 6 juillet 1996.

7. Difficile de ne pas voir ici un hommage aux «Jump photographs » qui firent la renommée du portraitiste Philippe Halsman dès la fin des années quarante. Ce procédé avait, par ailleurs, déjà 
donné une place à l'animal, qu'il s'agisse du chimpanzé J. Fred Muggs, vedette du Today Show de la NBC dans les années cinquante, ou, quelques années auparavant, des « chats volants » de la photographie intitulée Dali atomicus, célèbre portrait du peintre à l'œuvre, défiant la gravité.

8. Notamment The Attitudes of Animals in Motion en 1881 et Animal Locomotion en 1887.

9. Peut-on voir dans cette photographie un hommage au célèbre $\mathrm{Nu}$ descendant un escalier $n^{\circ} 2$ de Marcel Duchamp peint en 1912 ?

10. L'intérêt pour la symétrie, mais également pour les formes stabilisées, fixes, amène Wegman à user couramment des figures géométriques, voire à plier à une forme de géométrie le chien luimême. Ce sera par exemple le cas dans l'illustration du livre d'éducation Letters-NumbersPunctuation, en 1993, abécédaire composé entièrement à partir de ses chiens.

11. Procédé assimilable à une catachrèse, formulation par laquelle l'imaginaire de l'homme est à la fois fondu et figé.

12. On trouve également des animaux vêtus d'habits dans les illustrations de Grandville (dont s'inspirent les décors des assiettes publicitaires Royco, dans les années 20 à 40), dans les jouets mécaniques allemands du tout début du $\mathrm{xx}^{\mathrm{e}}$ siècle de Samuel Bing ou Philippe Vielmetter (faisant partie de la collection privée de Tomi Ungerer).

13. Notamment celui intitulé Zum Krisen-parteitag der SPD, publié en 1931, qui détourne le portrait d'un politicien flanqué d'une tête de tigre rugissant. Plus récemment, et grâce à l'image de synthèse, ou aux logiciels de retouche photographique, ce procédé perdure, et connaît même une nouvelle vigueur, allant s'amplifiant. On peut penser, entre autres, aux «Zoo portraits» du photographe Yago Partal, mais aussi, d'inspiration classique, aux peintures d'Olivia Beaumont et aux photomontages de Thierry Poncelet, aux multiples versions cinématographiques de La Planète des singes, ou encore à la campagne de la RATP, datant de 2013, et les illustrations du magazine Picnic, composées en 2010 par Alex Castro. L'exhaustivité ici serait difficile à atteindre et hors de propos, tant la liste des images usant de ce procédé est longue et ne cesse de croître.

14. William Wegman, Hansel and Gretel, trois panneaux polaroïd couleur, $24 \times 20 \mathrm{~cm}$ chacun, 2007.

15. Propos de l'artiste recueillis par Brigitte Ollier.

16. D'après Dominique Lestel, la véritable révolution qui reste à opérer est celle qui verra advenir l'animal-sujet : « Une culture se distingue de la société par la complexité des phénomènes sociaux mis en jeu et par la transformation de l'animal impliqué en sujet (...) Il n'y a pas de culture sans sujet (...) et il y a des espèces de sujets chez les animaux. C'est la révolution de l'éthologie contemporaine. $\mathrm{Qu}$ 'elle reste inaperçue, y compris chez la plupart des éthologues, est à verser sur le compte de l'ironie divine. » (Lestel, 2001 : 330)

17. À noter que les studios Disney ont produit en 1959 Quelle vie de chien!(The Shaggy Dog) de Charles Barton, d'après un roman de Felix Salten, dont le sujet est la transformation d'un jeune garçon en chien. Deux ans plus tard, le studio produira Bobby des Greyfriars (Greyfriars Bobby), un de Don Chaffey adapté d'une nouvelle d'Eleanor Atkinson publiée en 1912, à partir de faits réels liés à un Skye terrier écossais.

18. Auberger rappelle que même en revendiquant une objectivité scientifique, beaucoup de documentaristes animaliers peinent à penser l'animal en dehors de lui-même. (Auberger, 2007 : 142)

19. Il ne s'agit bien sûr ici que deux exemples parmi bien d'autres de ces animaux « intelligents » devenus de véritables stars du cinéma et de la télévision. Songeons à King-Kong le gorille géant, Cheeta la chimpanzé, Flipper le dauphin, Clarence la lionne, Beethoven le chien, Babe le cochon, et tous ceux qui n'eurent pas de nom comme par exemple les protagonistes du film L'Ours de Jean-Jacques Annaud...

20. Dans Le Problème de l'essence de l'homme chez Spinoza, le philosophe Julien Brusse « démontre qu'il ne peut y avoir d'essence de l'homme mais seulement d'un homme et il écarte l'idée selon laquelle le désir ou la raison pourraient suffire à la définir. » Chantal Jaquet, préface de Le Problème de l'essence de l'homme chez Spinoza (Brusse, 2009 : 7) 
21. Notons ici que d'autres artistes convoquent l'animal afin d'interroger l'espace de l'homme. Pierre Huyghe notamment, par un procédé totalement diffèrent, dans son court métrage Human mask, en 2014, laisse un singe, dont le visage est cette fois invisible, et le regard absent, s'approprier, de façon pernicieuse, la scène humaine.

22. Roman datant de 1955, porté à l'écran en 1962 par Stanley Kubrick.

23. Cf. Aristote, Politique et Éthique à Nicomaque.

24. À ce sujet, le cinéma dès son origine a donné le rôle principal à un chat; et surtout la première image animée a révélé, de manière fugace, ce regard animal. Il s'agit du Déjeuner du chat des frères Lumière ( $n^{\circ} 41$ du catalogue de vues Lumière), tourné en 1896, diffusé en 1897. Suivra, en 1898, Déjeuner des deux bébés et du minet ( $\mathrm{n}^{\circ} 959$ du catalogue).

25. Propos recueillis par Brigitte Ollier, op. cit.

26. De Fay, la chienne qui remplacera Man, et Batty, l'une de ses descendantes, il dresse des portraits sans équivoque : «D'abord, [Fay] n'ignorait pas qu'elle était un chien, et puis, elle était d'une beauté indescriptible: des yeux en amande, une élégance rare, très sensuelle, physiquement parfaite, avec des formes admirables. C'était une chienne d'un extrême raffinement et, à l'inverse de Man, infiniment sociable (...) Batty, l'une des deux filles de Fay, ressemble beaucoup à sa mère, mais elle n'atteint pas son raffinement. Fay était sérieuse, presque sévère, façon Joan Crawford. Batty, elle, s'adonne au comique. Elle est encore très juvénile et très sexy. C'est Lolita. » (Propos recueillis par Brigitte Ollier, op. cit.).

27. Selon le biologiste Yves Christen, « là où nous parlons d'animal, il faudrait toujours parler d'une personne animale singulière, qu'il s'agisse du chien Bobbie, du chimpanzé Nim ou de M. Durand. » (2013 : 11) Pour Wegman, percevoir le sujet avant de percevoir « le chien » constitue plus qu'une attitude intellectuelle: c'est une méthode artistique, une pratique autant qu'une habitude, une exigence, une visée.

28. Ère de la pierre taillée, les fossiles retrouvés datent de 10000 à 40000 avant JC. Le fait demeure, nous sommes d'accord pour affirmer que les chiens domestiqués et les loups modernes sont issus d'un ancêtre commun : le loup préhistorique. Les chiens ont sûrement débuté en tant que compagnons de chasse. En voyageant avec des humains nomades, ils auraient également agi comme source de protection.

29. Le chien aurait notamment représenté une réserve de force, une aide... Il est couramment admis que l'homme est devenu, pour une grande part, ce qu'il est, tel que nous nous connaissons, à l'époque où les loups sont devenus chiens.

30. D'ajouter: «Son zèle dominateur ne s'explique pas autrement que par la recherche de la domestication pour elle-même et pour l'image qu'elle renvoie d'un pouvoir sur la vie et les êtres. » (Ibid.)

31. Notion, entre autres, décrite dans Introduction to Animal Rights: Your Child or the Dog? (2000).

32. Aucune allusion à la bête élevée, abattue et consommée, souvent sans la moindre considération pour sa condition ou ce qu'elle représente. Pourtant, ce théâtre anthropomorphique finit, en négatif, par pointer l'incohérence d'une attitude capable de la plus vive empathie comme de la plus grande insouciance. Comment ne pas se dire que ces chiens, au pelage impeccable, choyés, observés, admirés, prennent une place bien trop importante, presque indécente, au vu des considérations si variables de l'humain au sujet des bêtes. Mais, ce que ressent le public de ces œuvres n'est pas cet attendrissement béat, cet infantilisme teinté d'un désir permanent de réification qui peut si facilement nous animer au contact de certains animaux. Car les chiens de Wegman sont avant tout des personnes dans lesquelles on ne peut pas, même subrepticement, ne pas se reconnaître.

33. Dont l'origine, encore incertaine, est sans doute à chercher à la fin du paléolithique. En témoignent les fossiles retrouvés datent de 10000 à 40000 avant JC. Le fait demeure, nous sommes d'accord pour affirmer que les chiens domestiqués et les loups modernes sont issus d'un ancêtre commun: le loup préhistorique. Les chiens ont sûrement débuté en tant que 
compagnons de chasse. En voyageant avec des humains nomades, ils auraient également agi comme source de protection.

34. Cf. Sigmund Freud, « Lettre du 21 septembre $1897 »$ (Freud, 1985).

35. Pour Penny Spikins, Holly Rutherford et Andy Needham notamment, elle serait le catalyseur du devenir homme. (Spikins, Rutherford, Needham, 2010)

\section{RÉSUMÉS}

Les chiens que William Wegman prend pour modèles - exclusivement des braques de Weimar sont devenus les acteurs d'une entreprise de figuration, constituant à eux seuls la signature et la renommée de l'artiste. Pris dans des dispositifs conçus suivant des règles du jeu sans cesse réinventées, l'animal représente dans l'œuvre de Wegman aussi bien un objet vivant, un comédien, un partenaire, qu'un interlocuteur, voire un alter ego. L'artiste, photographiant ou tournant des saynètes, confrontant les images des braques et des textes classiques ou personnels, reprend des situations qui n'appartiennent qu'à l'homme. Habillant souvent ses modèles et les plaçant dans des environnements qui sont également ceux de l'homme, parodiant des personnages connus, revisitant les genres les plus divers, Wegman joue avec ses chiens pour mieux se jouer de ses congénères. L'ironie, le clin d'œil, la dérision à l'œuvre lui permettent d'atteindre un large public, qui assiste, sur cette scène parallèle, à une relecture de l'humain, de ses codes et de ses travers.

The dogs that William Wegman uses as models - exclusively Weimaraners - became actors in a figurative enterprise, grantor of the artist's signature and fame. Taken in arrangements conceived according to rules of the constantly reinvented game, the animal represents a living object, as well as an actor, a partner, an interlocutor, and even an alter ego in Wegman's work. The artist, photographing or performing skits, confronting images of the hunting dogs and classical or personal texts, takes over situations that belong only to humans. Often dressing his models and placing them in environments that are also those of the humans, parodying known characters, revisiting the most diverse genres, Wegman plays with his dogs to better mock his fellow. The irony, the wink, the derision at work allow him to reach a wide audience, which attends, on this parallel scene, a reinterpretation of the human, of its codes and faults.

\section{INDEX}

Mots-clés : chien, jeu, ironie, genres, anthropomorphisme

Keywords : dog, game, irony, genres, anthropomorphism

\section{AUTEUR}

\section{VINCENT LECOMTE}

CIEREC/Université Jean Monnet de Saint-Étienne

vincent.lecomte[at]univ-st-etienne.fr 